International Review of Research in Open and Distributed Learning Volume 23, Number 1

February - 2022

\title{
Editorial - Volume 23, Issue 1
}

\section{Special Issue: Al e-Learning and Online Curriculum}

Ting-Chia Hsu', Hal Abelson², and Natalie Lao ${ }^{3}$

${ }^{1}$ Department of Technology Application and Human Resource Development, National Taiwan Normal University, Taiwan;

${ }^{2}$ Computer Science and Artificial Intelligence Lab, Massachusetts Institute of Technology, USA; ${ }^{3}$ Department of Electrical

Engineering and Computer Science, Massachusetts Institute of Technology, USA

This special issue was created due to the growing impact of artificial intelligence (AI) in education. As such, we collected seven articles for this issue that captured this interesting phenomenon. Each article underwent a double-blind peer review to ensure that only the most relevant and highest quality papers made this special issue.

The trend of AI in online-learning research is important. From Hwang, Tu, and Tang's review study, we can see that the online learning system interactions, which can facilitate personalized, adaptive, and collaborative learning, became a focus in recent years. Regarding implementation, advancements in hardware processing speeds, networking, and machine learning algorithms are assisting in the advancement of teaching and learning. For example, AI techniques can be used to predict failure in online learning, the detection of a student's risk of dropping out, or the prediction of student course satisfaction scores. Tzeng, Lee, Huang, Huang, and Lai's empirical study revealed that their early warning system, using the fifth-week model for online learning, successfully predicted student performance with an accuracy exceeding 83\%. Rodríguez, Guerrero-Roldán, Baneres, and Karadeniz employed a nudging intervention mechanism based on the AI technique into positively affecting the learners' performance and dropout reduction in online learning. However, Tzeng, Lee, Huang, Huang, and Lai used deep learning to assess the student experience with MOOCs and constructed a deep learning model to accurately predict student satisfaction without relying on questionnaire responses.

The global COVD-19 pandemic is forcing more and more schools to experiment with online education. This puts a premium on curricula and tools that can lead to effective distance learning and also raises a host of additional considerations for educational technology research and practice. In Araka, Oboko, Maina, and Gitonga's review of Educational Data Mining (EDM) techniques and how they have been widely applied in online learning, they discovered an optimal EDM algorithm, Agglomerative Hierarchical, for identifying the levels of self-regulated learning profiles in online learning environments. To connect information by using AI techniques is also valuable for personalized learning. To overcome the huge amount of information in search engines, Cheng, Cheng, and Huang developed an Internet articles retrieval agent combined with dynamic associative concept maps, in order to help students find intended articles in their searches.

Regarding curriculum, the growing influence of AI on society makes it important for students to understand 
the fundamentals of AI and the application and use of AI in daily life. Cloud computing, open databases, and everything from application programming interfaces to large machine-learning services are making it possible for even young children to experiment with applications that only five years ago were only accessible to certain researchers. For example, the conversational AI learning platform was developed for MIT's App Inventor in 2019. Hsu, Abelson, and Van Brummelen employed this instructional tool to a junior high school in Taiwan and the results showed that the interactive learning tool for programming the AI application contributed to an enhancement of women in technology. The learning effectiveness of female students was significantly better than that of male students regardless of whether they used an experiential learning approach or a conventional approach. These curricula and tools can have enormous worldwide influence when they are shared open-access and worldwide so that everyone can build on and improve them.

\section{Athabasca} University

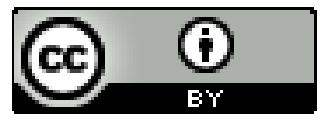

